\title{
REVIEW
}

\section{Is consuming yoghurt associated with weight management outcomes? Results from a systematic review}

\author{
J Eales ${ }^{1}$, I Lenoir-Wijnkoop ${ }^{2}$, S King ${ }^{1}$, H Wood ${ }^{1}$, FJ Kok ${ }^{3}$, R Shamir ${ }^{4}$, A Prentice ${ }^{5}$, M Edwards' ${ }^{1}$, J Glanville ${ }^{1}$ and RL Atkinson ${ }^{6}$
}

BACKGROUND: Yoghurt is part of the diet of many people worldwide and is commonly recognised as a 'health food'. Epidemiological studies suggest that yoghurt may be useful as part of weight management programs. In the absence of comprehensive systematic reviews, this systematic review investigated the effect of yoghurt consumption by apparently healthy adults on weight-related outcomes.

METHODS: An extensive literature search was undertaken, as part of a wider scoping review, to identify yoghurt studies. A total of 13631 records were assessed for their relevance to weight-related outcomes.

RESULTS: Twenty-two publications were eligible according to the review protocol. Cohort studies $(n=6)$ and cross-sectional studies $(n=7)$ all showed a correlation between yoghurt and lower or improved body weight/composition. Six randomised controlled trials (RCTs) and one controlled trial had various limitations, including small size and short duration. One RCT showed significant effects of yoghurt on weight loss, but was confounded by differences in calcium intake. One trial showed nonsignificant weight gain and the remaining five trials showed nonsignificant weight losses that were greater in yoghurt consumers.

CONCLUSIONS: Yoghurt consumption is associated with lower body mass index, lower body weight/weight gain, smaller waist circumference and lower body fat in epidemiological studies. RCTs suggest weight reduction effects, but do not permit determination of a cause-effect relationship. Well-controlled, adequately powered trials in research and community settings appear likely to identify a modest but beneficial effect of yoghurt consumption for prevention of weight gain and management of obesity. The ready availability of yoghurt (a nutrient-dense food) and its ease of introduction to most diets suggests that educating the public to eat yoghurt as part of a balanced and healthy diet may potentially contribute to improved public health. Future carefully designed RCTs could provide proof of principle and large community-based studies could determine the practical impact of yoghurt on body weight/composition.

International Journal of Obesity (2016) 40, 731-746; doi:10.1038/ijo.2015.202

\section{INTRODUCTION}

Conventional (non-probiotic) yoghurt is a common feature of the food consumption patterns of people worldwide. The beneficial health effects of yoghurt have been the subject of investigation for over a century using observational and experimental studies. ${ }^{1,2}$ The Codex Alimentarius International Food Standards define yoghurt as a form of fermented milk that contains symbiotic cultures of Streptococcus thermophilus (sp) subsp. salivarius and Lactobacillus delbrueckii subsp. bulgaricus. ${ }^{3}$ Conventional yoghurt must contain milk protein (minimum $2.7 \%$ ), milk fat $(<15 \%)$, lactic acid (minimum 0.6\%) and microorganisms in the proper proportions. ${ }^{3}$ It does not have the additional further strains of probiotic bacteria, ${ }^{4}$ such as Bifidobacterium lactis or Lactobacillus acidophilus, which feature in probiotic yoghurt. Probiotic yoghurt has been the subject of much primary research and systematic reviews over the past two decades, but the evidence picture for conventional yoghurt has not been extensively investigated.

The efficacy of yoghurt has been investigated in a wide range of separate and overlapping outcomes including weight-related outcomes, ${ }^{5}$ type 2 diabetes, ${ }^{6}$ cardiovascular disease risk, ${ }^{7}$ the risk of cancer, ${ }^{8}$ gastrointestinal health, ${ }^{9}$ diarrhoea symptoms, ${ }^{10}$ malnutrition ${ }^{11}$ and overall mortality. ${ }^{12}$ Systematic reviews (some involving meta-analysis) have been conducted in relation to only a few of these outcomes. Their limited results suggest that consuming yoghurt may reduce the risk of developing type 2 diabetes, ${ }^{13-15}$ but may have no effect on the risk of developing colorectal cancer, ${ }^{16}$ hypertension ${ }^{17}$ or overall mortality. ${ }^{18} \mathrm{~A}$ systematic review of the effects of conventional yoghurt consumption on weight-related outcomes has not been published, to our knowledge. In an increasingly obese population, it would be of value for public health strategies to know whether an inexpensive and commonly consumed foodstuff can assist in weight-related outcomes. This review was conducted to investigate the effectiveness of standard yoghurt on a range of weightrelated outcomes in the general, apparently healthy, adult population. The review was also designed to identify data on effectiveness in prespecified subgroups such as people at risk of diabetes, and people from different ethnic groups.

\section{METHODS}

This review was conducted using systematic review methods, involving the systematic and transparent identification, selection,

\footnotetext{
${ }^{1}$ York Health Economics Consortium, University of York, York, UK; ${ }^{2}$ Utrecht University, Utrecht, The Netherlands; ${ }^{3}$ Wageningen University, Wageningen, The Netherlands; ${ }^{4}$ Institute for Gastroenterology, Nutrition and Liver Diseases, Schneider Children's Medical Center, Sackler Faculty of Medicine, Tel-Aviv University, Tel-Aviv, Israel; ${ }^{5}$ London School of

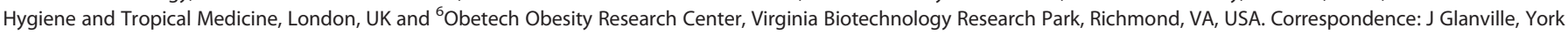
Health Economics Consortium, Enterprise House, Innovation Way, University of York, Heslington, York YO10 5NQ, UK. 
extraction and synthesis of studies relevant to the research question. ${ }^{19}$ The review is reported in accordance with the Preferred Reporting Items for Systematic Reviews and MetaAnalyses (PRISMA) reporting guidance. ${ }^{20}$ The completed PRISMA checklist is provided in the Supplementary File. Details of the protocol for this systematic review were registered on PROSPERO and can be accessed at www.crd.york.ac.uk/PROSPERO/display_ record.asp?ID=CRD42014013883.

\section{Review question}

This review aimed to investigate the effects of yoghurt containing the symbiotic cultures Streptococcus thermophilus (sp) subsp. salivarius and Lactobacillus delbrueckii subsp. bulgaricus on weightrelated outcomes in the general, apparently healthy, adult population. The eligibility criteria, used to select the studies to be included in the review, were developed from the review question. They are summarised below and presented in detail in the Supplementary File.

\section{Participants}

Eligible studies assessed apparently healthy adults (18-65 years) or mixed populations where separate results were presented for the 18-65 years age group.

Studies reporting data for subgroups of the general population who were at high risk of weight change were eligible for inclusion, for example, people with insulin resistance, diabetes or obesity (body mass index (BMI), between 30 and $40 \mathrm{~kg} \mathrm{~m}^{-2}$ ).

Studies in specific populations were excluded because we were interested in the effects in a generally healthy, normal population. Therefore, people with severe or morbid obesity $\left(B M I \geqslant 40.00 \mathrm{~kg} \mathrm{~m}^{-2}\right.$ ), people with a single specific disease or symptom and people training for or undertaking physical activity at a professional level were excluded.

\section{Interventions}

Eligible studies investigated consumption of yoghurt (all flavours and fat contents were eligible) containing the standard symbiotic cultures. Studies of yoghurt in combination with another substance (e.g. added protein/vitamins/fats) were not eligible as yoghurt was likely being used as a carrier substance for an active ingredient and hence was not the focus of the investigation. Studies of probiotic yoghurt, fermented milk or formula, kefir or kumys were not eligible for inclusion.

\section{Comparators}

Eligible studies had to compare yoghurt with at least one of the following:

1. low or no yoghurt consumption;

2. placebo (e.g. yoghurt-like products not including live bacteria);

3. non-yoghurt substances (e.g. milk);

4. non-yoghurt interventions (e.g. nutritional counselling).

\section{Outcomes}

Eligible studies had to assess the interventions in relation to at least one weight-related outcome: body weight, BMI, percent body fat, percent lean body mass, waist circumference (WC) or composite measures of the above weight-related outcomes.

\section{Study types}

Randomised and quasi-randomised controlled trials (RCTs), controlled clinical trials, comparative observational studies (including cross-sectional studies, cohort studies and case-control studies) and systematic reviews were eligible for the review. Studies published as abstracts, conference presentations or unpublished reports were eligible.

\section{Literature search}

An extensive literature search was undertaken, as part of a wider scoping review to identify yoghurt studies published in the health sciences. Search terms were based on synonyms for the intervention (yoghurt) and the full searches for all resources are listed in the Supplementary File. The searches were not limited by date or language. Searches were undertaken in 39 databases and websites in October 2014. The reference lists of relevant reviews, trials and studies were checked to identify any further studies.

\section{Study selection}

Records identified by the searches were first assessed for relevance by one reviewer (ME) to rapidly remove obviously irrelevant records. Record selection was then undertaken by two reviewers independently, based on information in the title and abstract of records. Where possible, the full documents of possibly relevant studies were obtained and were assessed for relevance by one reviewer (JE) and checked by a second independent reviewer (SK). In the latter two stages, discrepancies were resolved through discussion and, where necessary, by consulting a third reviewer (JG). Studies that were considered ineligible at this stage were recorded in a table with reasons for exclusion (see Supplementary File). Studies that were unobtainable are included in the Supplementary File. The number of studies identified by the search and excluded at various stages are shown in a record selection diagram (Figure 1). ${ }^{20}$

\section{Data extraction and quality assessment}

One researcher (JE) extracted the data and study information from the eligible studies into Excel, and a second researcher (DC or SK) checked the extraction. Any discrepancies were resolved through discussion or by consulting a third reviewer (JG). Data were extracted from abstracts when no full document was available. Data extraction elements are listed in the Supplementary File. When required information was not presented in a document, it was requested from the study authors.

Quality assessment of the internal and external validity of eligible studies was undertaken using the Cochrane Risk of Bias tool for RCTs, controlled trials and cross-over trials, ${ }^{19}$ the Centre for Reviews and Dissemination tool for cohort studies ${ }^{21}$ and the Newcastle-Ottawa Quality Assessment Scale adapted for crosssectional studies (see Supplementary File for details). ${ }^{22}$ Quality assessment was conducted by one researcher (JE) and checked by a second researcher (DC or SK). Any disagreements were discussed between the reviewers.

\section{Data synthesis}

Following an assessment of the comparability of their populations, interventions and outcomes, only a few RCTs provided data suitable for meta-analysis for some outcomes. Lack of raw data reporting was another factor preventing the meta-analysis of cohort and cross-sectional studies. Meta-analysis was conducted using a random-effects model as the RCTs were somewhat heterogeneous (e.g. different ethnic composition of study groups). Because the number of studies was small, the analyses were also conducted using a fixed-effect model.

Heterogeneity was assessed using the $x^{2}$ and $l^{2}$ statistics, where an $P^{2}$ of $0-25 \%$ represents no heterogeneity, $25-50 \%$ represents moderate heterogeneity, $50-75 \%$ represents substantial heterogeneity and $75-100 \%$ represents considerable heterogeneity. The level of statistical significance for effect size and summary effect size was set at $P<0.05$. 


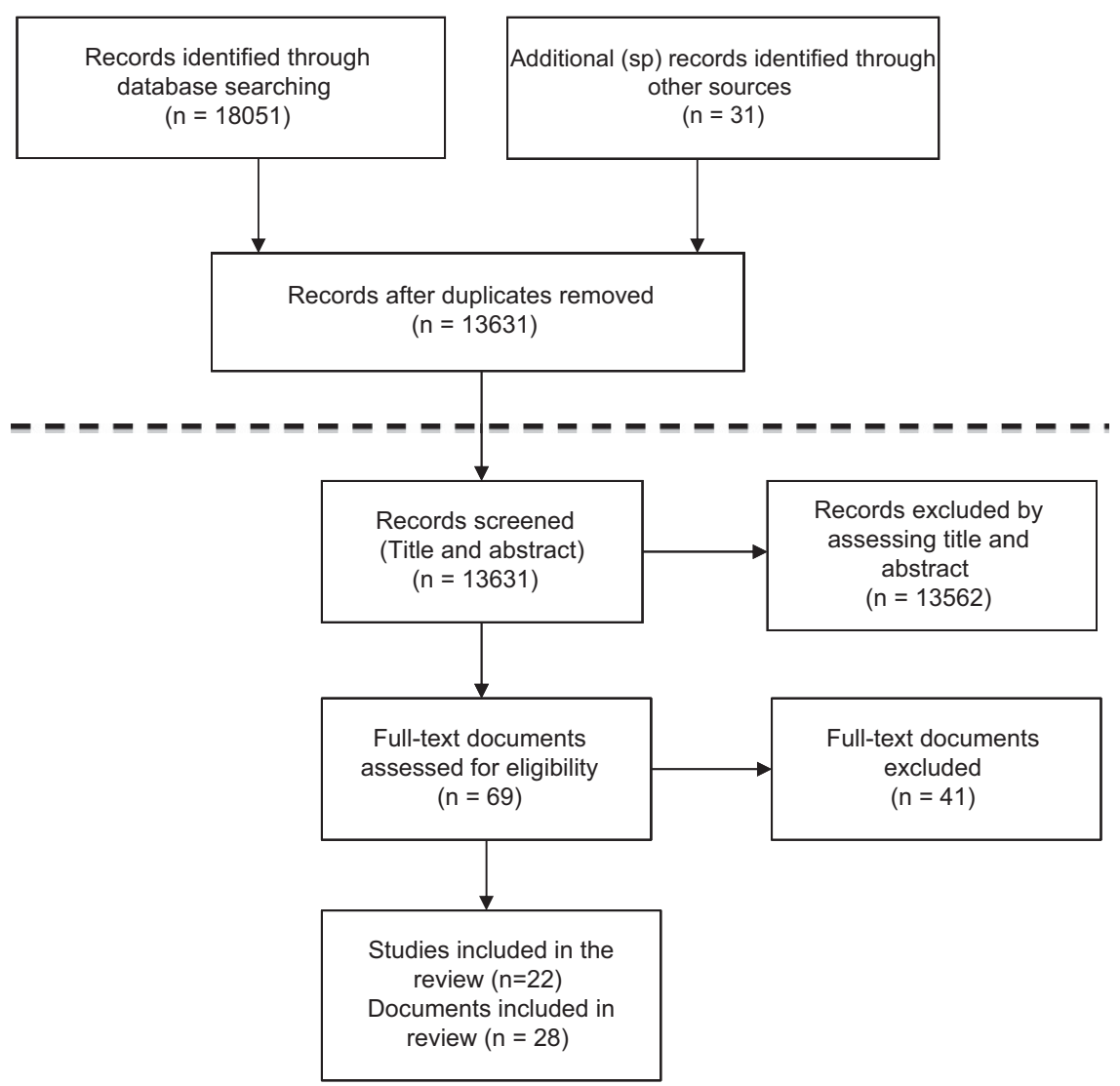

Figure 1. Record selection process. Above the dashed line indicates records retrieved from a wide scoping search for studies of yoghurt in the health science literature. Below the dashed line indicates where study selection criteria for this systematic review (yoghurt for weight-related outcomes were applied.

Raw data provided by study authors was used for the mean and s.d. of weight-related outcomes. These data were pooled to estimate the combined effect size (mean difference with $95 \%$ confidence intervals $(\mathrm{Cls}))$ across studies for:

- Change in body weight in participants with an energyrestricted diet.

- Yoghurt vs control (three RCTs).

- Change in waist circumference (WC) in participants with an energy-restricted diet.

- Yoghurt vs control (two RCTs).

- Change in body fat in participants with an energyrestricted diet.

- Yoghurt vs control (two RCTs).

Where standard error of the mean was reported, rather than standard deviation, the standard deviation was calculated using the formula:

$$
\mathrm{SD}=\mathrm{SEM} * \sqrt{N}
$$

where s.e.m. is the standard error of the mean and $N$ the sample size.

The results from the meta-analyses are graphically presented as forest plots. All analyses were conducted using RevMan version 5.3. Where possible, a sensitivity analysis was undertaken, excluding studies with a high risk of bias.

\section{RESULTS}

Search results

A total of 13631 records (after deduplication) were identified by extensive searches (see Supplementary File for details). Of these, 69 full documents were assessed for relevance to the topic of yoghurt for weight-related outcomes (Figure 1). Two reports published as abstracts were potentially relevant, but were excluded as further details were not provided by the authors. $^{23,24}$ Twenty-two studies (reported in 28 documents) were included in the review (Table 1$).^{25-45}$

\section{Characteristics of included studies}

Study characteristics are provided in Table 1. There were six RCTs, one controlled trial (CT), two cross-over studies, one prospective cohort study, five retrospective population-based cohort studies and seven cross-sectional studies. The studies were carried out in the USA $(n=14)$, Korea $(n=1)$, Malaysia $(n=1)$, Spain $(n=1)$, Australia $(n=1)$, Canada $(n=2)$, France $(n=1)$ and one study did not report the location. Where reported, study dates ranged from $1979^{32}$ to September 2010. ${ }^{34}$

Generally, RCTs, cross-over trials and the prospective cohort study had the smallest study sizes. RCTs generally had the shortest duration (range: 14 days $^{25}$ to 24 weeks $^{26}$ ).

Cross-sectional and population-based cohort study numbers ranged from 72 participants ${ }^{45}$ to 14500 participants, ${ }^{41}$ with an outlying large study of 120877 participants. ${ }^{36}$ Cohort study duration ranged from 5.9 years ${ }^{35}$ to 12.9 years. ${ }^{39}$ 


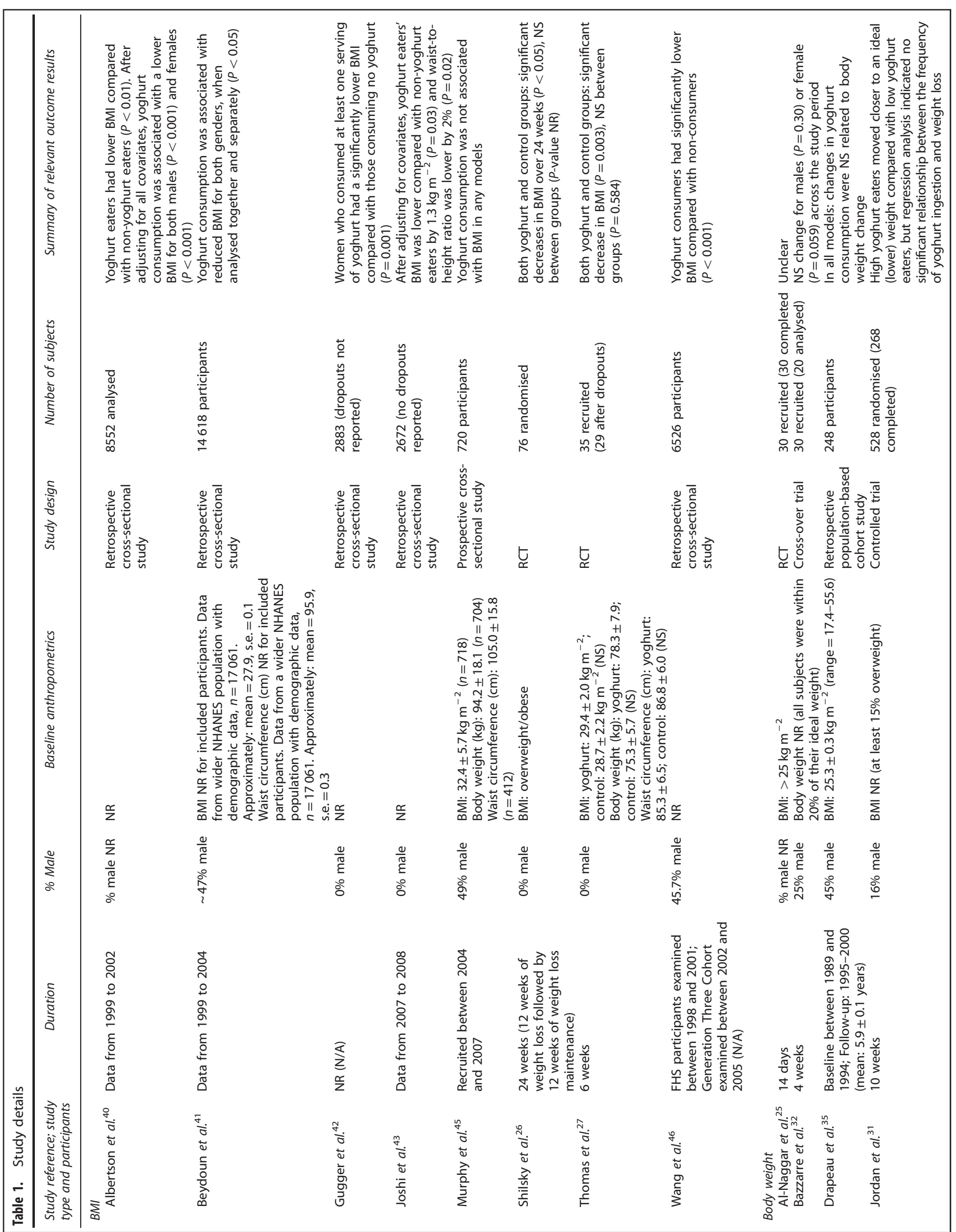




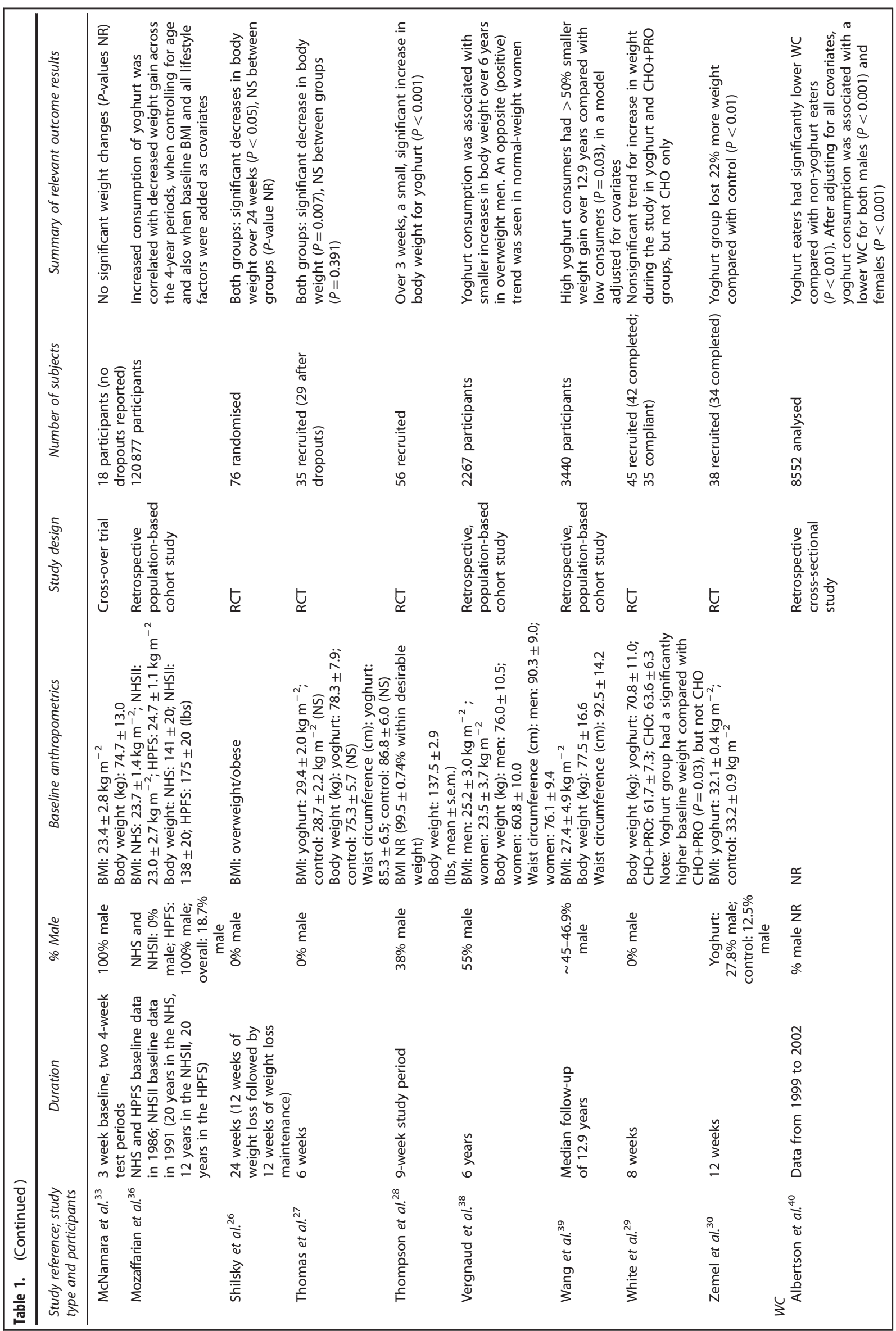




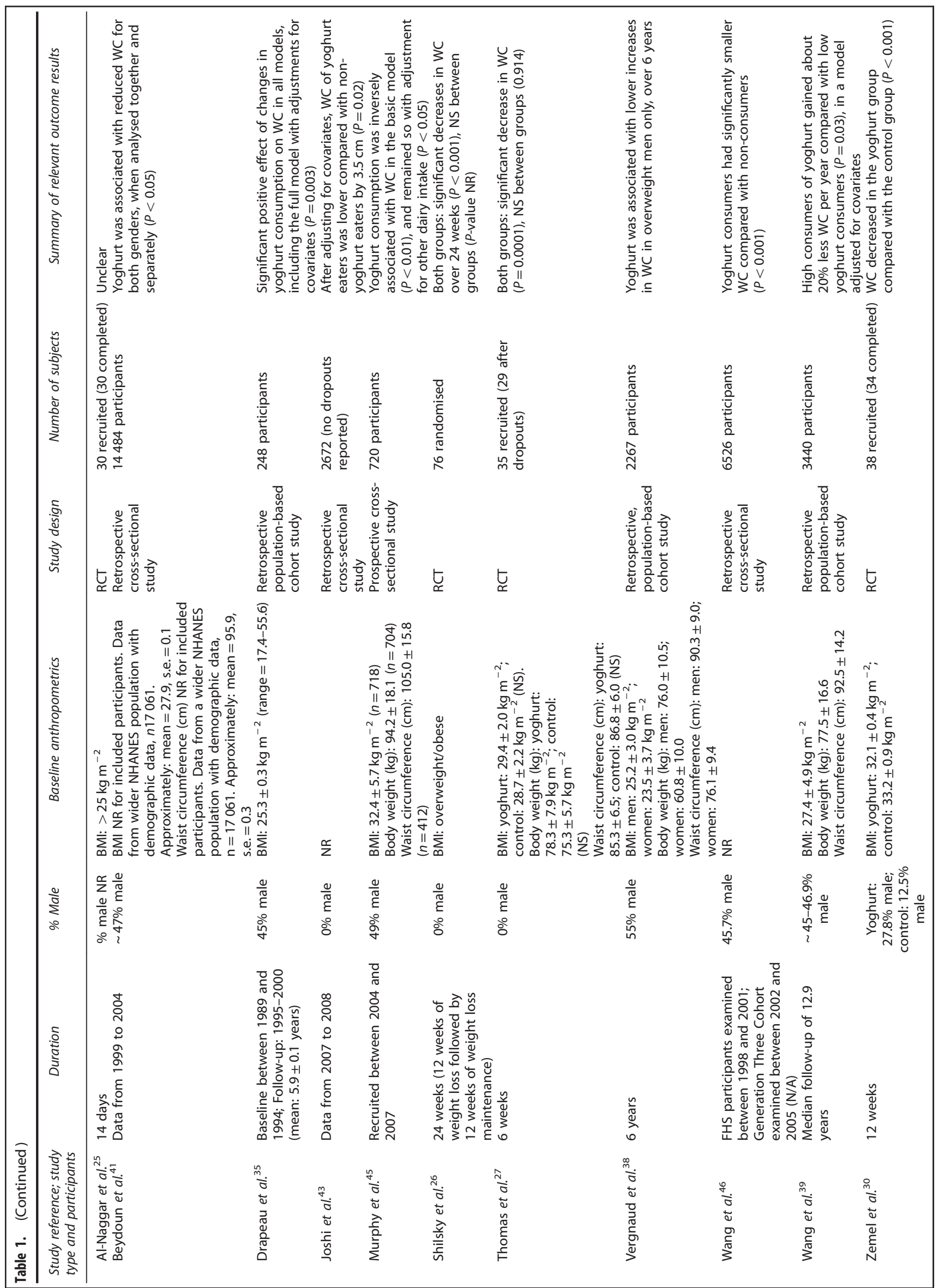




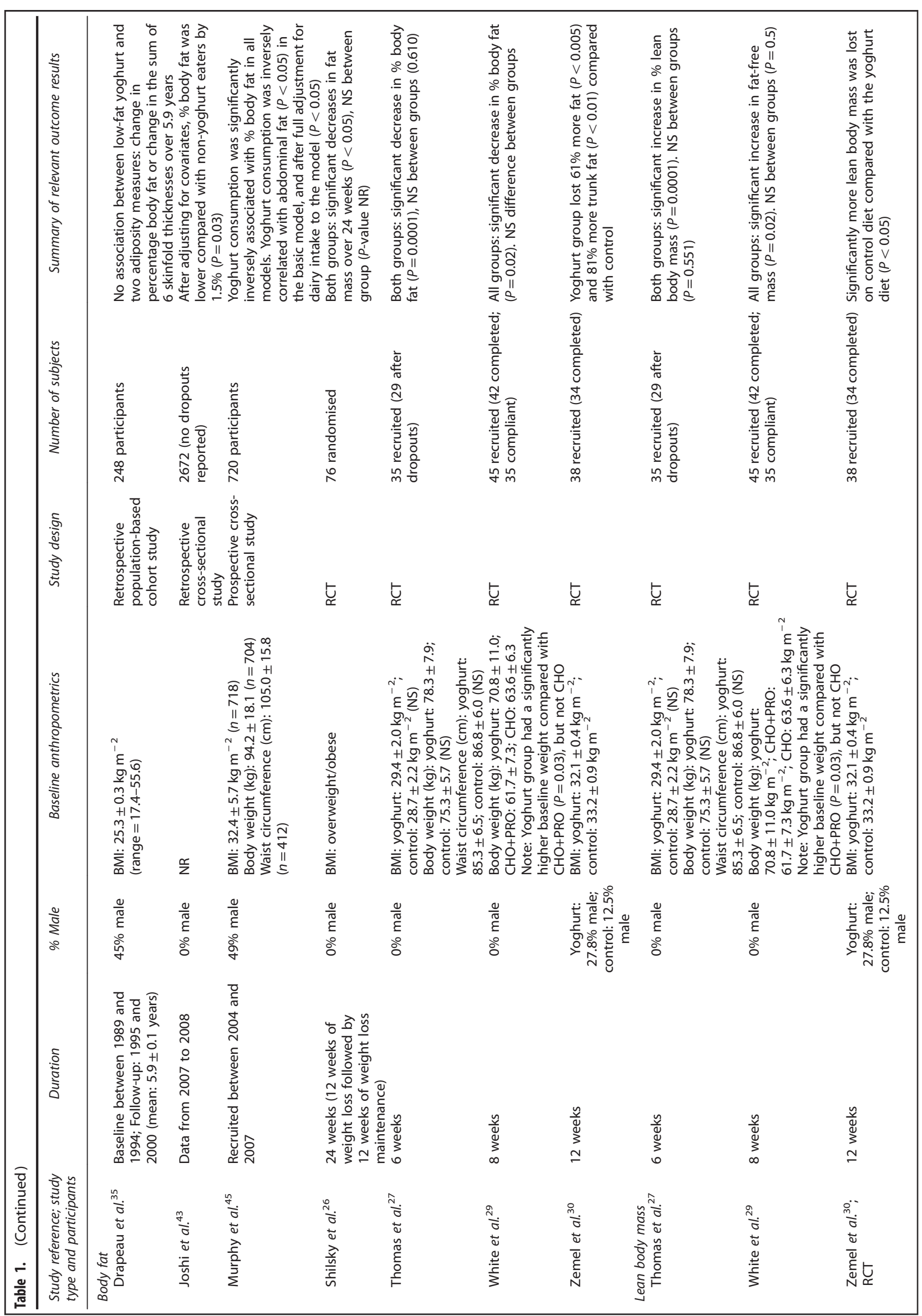




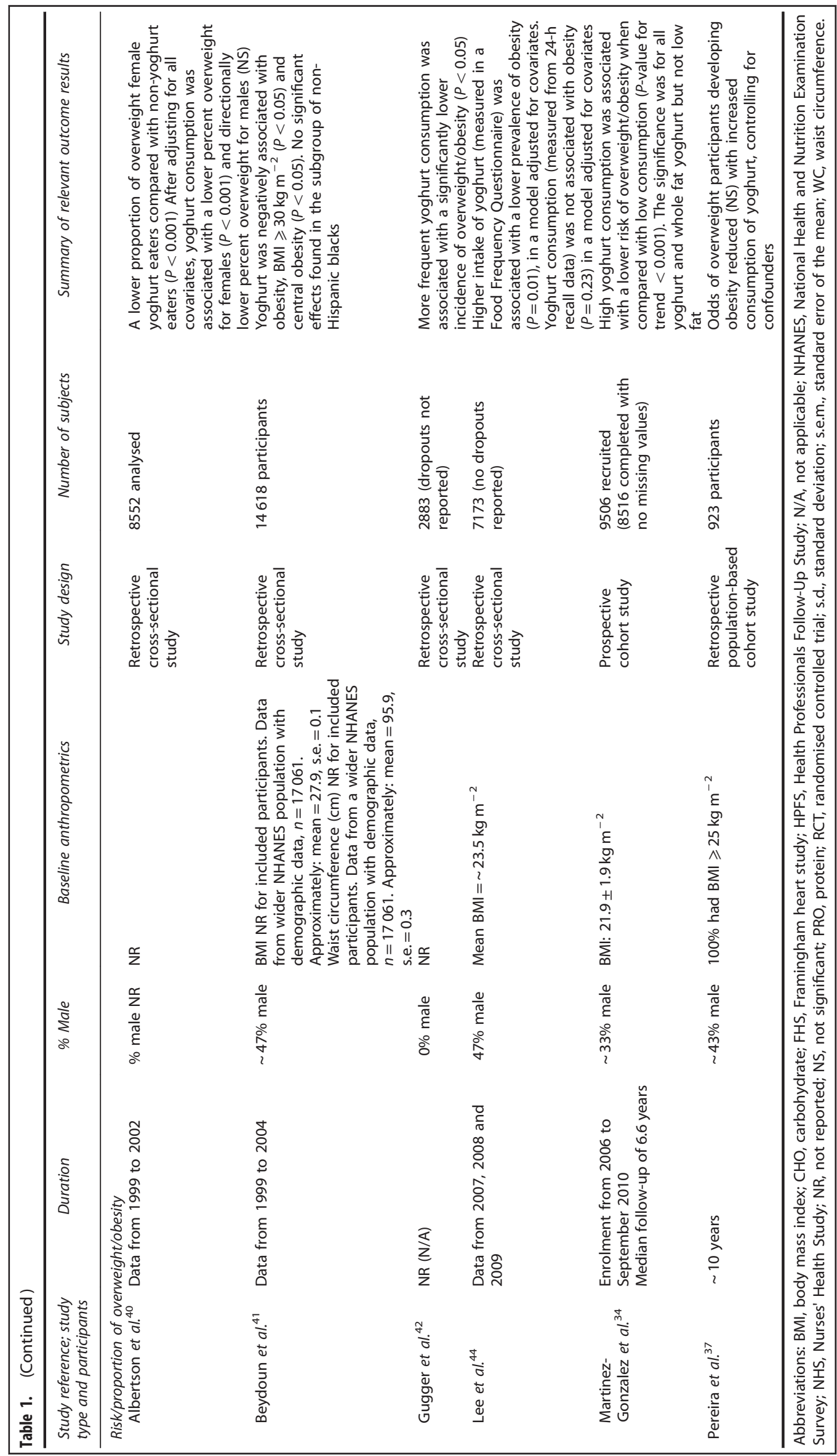


Table 2. Summary of included studies

Study reference; study type and participants

Summary of relevant outcome results

$B M I$

Albertson et al. ${ }^{40}$

Retrospective cross-sectional study; 8552 analysed

Beydoun et al..$^{41}$

Retrospective cross-sectional study; 14618 participants

Gugger et al. ${ }^{42}$

Retrospective cross-sectional study; 2883 (dropouts not reported)

Joshi et al. ${ }^{43}$

Retrospective cross-sectional study; 2672 (no dropouts reported)

Murphy et $a l^{45}$

Prospective cross-sectional study; 720 participants

Shilsky et al. ${ }^{26}$

$\mathrm{RCT} ; 76$ randomised

Thomas et al. $^{27}$

$\mathrm{RCT} ; 35$ recruited (29 after dropouts)

Wang et $a l^{46}$

Retrospective cross-sectional study; 6526 participants

Body weight

Al-Naggar et al. ${ }^{25}$

$\mathrm{RCT} ; 30$ recruited (30 completed)

Bazzarre et al. ${ }^{32}$

Cross-over trial; 30 recruited (20 analysed)

Drapeau et al. ${ }^{35}$

Retrospective population-based cohort study; 248 participants

Jordan et al. $^{31}$

Controlled trial; 528 randomised (268 completed)

McNamara et al. ${ }^{33}$

Cross-over trial; 18 participants (no dropouts reported)

Mozaffarian et al. ${ }^{36}$

Retrospective population-based cohort study; 120877

participants

Shilsky et al. $^{26}$

$\mathrm{RCT} ; 76$ randomised

Thomas et al. ${ }^{27}$

$\mathrm{RCT} ; 35$ recruited (29 after dropouts)

Thompson et al. ${ }^{28}$

RCT; 56 recruited

Vergnaud et $a l^{38}$

Retrospective, population-based cohort study; 2267 participants

Wang et al. ${ }^{39}$

Retrospective population-based cohort study; 3440 participants White et al. ${ }^{29}$

$\mathrm{RCT}$; 45 recruited (42 completed; 35 compliant)

Zemel et al. ${ }^{30}$

$\mathrm{RCT} ; 38$ recruited (34 completed)

WC

Albertson et al. ${ }^{40}$

Retrospective cross-sectional study; 8552 analysed

Al-Naggar et al. $^{25}$

$\mathrm{RCT} ; 30$ recruited (30 completed)

Beydoun et al. ${ }^{41}$

Retrospective cross-sectional study; 14484 participants

Drapeau et al. ${ }^{35}$

Retrospective population-based cohort study; 248 participants Joshi et al. $^{43}$

Retrospective

Murphy et al. ${ }^{45}$

Prospective cross-sectional study; 720 participants

Shilsky et al. ${ }^{26}$

$\mathrm{RCT} ; 76$ randomised

Thomas et al. ${ }^{27}$

$\mathrm{RCT} ; 35$ recruited (29 after dropouts)
Yoghurt eaters had lower BMI compared with non-yoghurt eaters $(P<0.01)$. After adjusting for all covariates, yoghurt consumption was associated with a lower BMI for both males $(P<0.001)$ and females $(P<0.001)$

Yoghurt consumption was associated with reduced BMl for both genders,

when analysed together and separately $(P<0.05)$

Women who consumed at least one serving of yoghurt had a significantly

lower BMI compared with those consuming no yoghurt $(P=0.001)$

After adjusting for covariates, yoghurt eaters' BMI was lower compared with

non-yoghurt eaters by $1.3 \mathrm{~kg} \mathrm{~m}^{-2}(P=0.03)$ and waist-to-height ratio was

lower by $2 \%(P=0.02)$

Yoghurt consumption was not associated with BMI in any models

Both yoghurt and control groups: significant decreases in BMI over 24 weeks $(P<0.05)$, NS between groups $(P$-value NR)

Both yoghurt and control groups: significant decrease in BMI $(P=0.003)$,

NS between groups $(P=0.584)$

Yoghurt consumers had significantly lower BMI compared with

non-consumers $(P<0.001)$

Unclear

NS change for males $(P=0.30)$ or female $(P=0.059)$ across the study period

In all models: changes in yoghurt consumption were NS related to body weight change

High yoghurt eaters moved closer to an ideal (lower) weight compared to low yoghurt eaters, but regression analysis indicated no significant relationship between the frequency of yoghurt ingestion and weight loss No significant weight changes ( $P$-values NR)

Increased consumption of yoghurt was correlated with decreased weight gain across the 4-year periods, when controlling for age and also when baseline $\mathrm{BMI}$ and all lifestyle factors were added as covariates

Both groups: significant decreases in body weight over 24 weeks $(P<0.05)$, NS between groups ( $P$-value NR)

Both groups: significant decrease in body weight $(P=0.007)$, NS between groups $(P=0.391)$

Over 3 weeks, a small, significant increase in body weight for yoghurt $(P<0.001)$

Yoghurt consumption was associated with smaller increases in body weight over 6 years in overweight men. An opposite (positive) trend was seen in normal-weight women

High yoghurt consumers had $>50 \%$ smaller weight gain over 12.9 years compared with low consumers $(P=0.03)$, in a model adjusted for covariates Nonsignificant trend for increase in weight during the study in yoghurt and $\mathrm{CHO}+\mathrm{PRO}$ groups, but not $\mathrm{CHO}$ only

Yoghurt group lost $22 \%$ more weight compared with control $(P<0.01)$

Yoghurt eaters had significantly lower WC compared with non-yoghurt eaters $(P<0.01)$. After adjusting for all covariates, yoghurt consumption was associated with a lower WC for both males $(P<0.001)$ and females $(P<0.001)$ Unclear

Yoghurt was associated with reduced WC for both genders, when analysed together and separately $(P<0.05)$

Significant positive effect of changes in yoghurt consumption on WC in all models, including the full model with adjustments for covariates $(P=0.003)$ After adjusting for covariates, WC of yoghurt eaters was lower compared with non-yoghurt eaters by $3.5 \mathrm{~cm}(P=0.02)$

Yoghurt consumption was inversely associated WC in the basic model $(P<0.01)$, and remained so with adjustment for other dairy intake $(P<0.05)$ Both groups: significant decreases in WC over 24 weeks $(P<0.001)$, NS between groups ( $P$-value NR)

Both groups: significant decrease in WC $(P=0.0001)$, NS between groups (0.914) 
Table 2. (Continued)

Study reference; study type and participants

Summary of relevant outcome results

Vergnaud et al. ${ }^{38}$

Retrospective, population-based cohort study; 2267 participants Wang et al. ${ }^{46}$

Retrospective cross-sectional study; 6526 participants

Wang et al. ${ }^{39}$

Retrospective population-based cohort study; 3440 participants Zemel et al. ${ }^{30}$

$\mathrm{RCT} ; 38$ recruited (34 completed)

over 6 years

Yoghurt consumers had significantly smaller WC compared with non-

consumers $(P<0.001)$

High consumers of yoghurt gained about $20 \%$ less WC per year compared with low yoghurt consumers $(P=0.03)$, in a model adjusted for covariates WC decreased in the yoghurt group compared with the control group $(P<0.001)$

Body fat

Drapeau et al. ${ }^{35}$

Retrospective population-based cohort study; 248 participants

Joshi et al. ${ }^{43}$

No association between low-fat yoghurt and two adiposity measures: change in percentage body fat or change in the sum of 6 skinfold thicknesses over 5.9 years

Retrospective cross-sectional study; 2672 (no dropouts reported) Murphy et al. ${ }^{45}$

Prospective cross-sectional study; 720 participants

After adjusting for covariates, \% body fat was lower than non-yoghurt eaters

by $1.5 \%(P=0.03)$

Yoghurt consumption was significantly inversely associated with \% body fat in all models. Yoghurt consumption was inversely correlated with abdominal fat $(P<0.05)$ in the basic model, and after full adjustment for dairy intake to the model $(P<0.05)$

Shilsky et al. ${ }^{26}$

$\mathrm{RCT} ; 76$ randomised

Thomas et al. ${ }^{27}$

RCT; 35 recruited (29 after dropouts)

White et al. ${ }^{29}$

RCT; 45 recruited (42 completed; 35 compliant)

Zemel et al. $^{30}$

$\mathrm{RCT} ; 38$ recruited (34 completed)

Both groups: significant decreases in fat mass over 24 weeks $(P<0.05)$, NS between group ( $P$-value NR)

Both groups: significant decrease in \% body fat $(P=0.0001)$, NS between groups (0.610)

All groups: significant decrease in $\%$ body fat $(P=0.02)$. NS difference between groups

Yoghurt group lost $61 \%$ more fat $(P<0.005)$ and $81 \%$ more trunk fat $(P<0.01)$ compared with control

Lean body mass

Thomas et al. ${ }^{27}$

RCT; 35 recruited (29 after dropouts)

White et al. ${ }^{29}$

$\mathrm{RCT} ; 45$ recruited (42 completed; 35 compliant)

Zemel et al. ${ }^{30}$

$\mathrm{RCT} ; 38$ recruited (34 completed)

Both groups: significant increase in $\%$ lean body mass $(P=0.0001)$, NS between groups $(P=0.551)$

All groups: significant increase in fat-free mass $(P=0.02)$. NS between groups $(P=0.5)$

Significantly more lean body mass was lost on control diet compared with the yoghurt diet $(P<0.05)$

Risk/proportion of overweight/obesity

Albertson et al. ${ }^{40}$

Retrospective cross-sectional study; 8552 analysed

A lower proportion of overweight female yoghurt eaters compared with nonyoghurt eaters $(P<0.001)$. After adjusting for all covariates, yoghurt consumption was associated with a lower percent overweight for females $(P<0.001)$ and directionally lower percent overweight for males (NS) Yoghurt was negatively associated with obesity, $\mathrm{BMI} \geqslant 30(P<0.05)$ and

Beydoun et al. ${ }^{41}$

Retrospective cross-sectional study; 14618 participants central obesity $(P<0.05)$. No significant effects found in the subgroup of non-Hispanic blacks

Gugger et al. ${ }^{42}$ More frequent yoghurt consumption was associated with a significantly Retrospective cross-sectional study; 2883 (dropouts not reported) lower incidence of overweight/obesity $(P<0.05)$ Lee et al. ${ }^{44}$ Higher intake of yoghurt (measured in a Food Frequency Questionnaire) was Retrospective cross-sectional study; 7173 (no dropouts reported)

Martinez-Gonzalez et al. ${ }^{34}$

Prospective cohort study; 9506 recruited (8516 completed with no missing values)

Pereira et al. ${ }^{37}$

Retrospective population-based cohort study; 923 participants

associated with a lower prevalence of obesity $(P=0.01)$, in a model adjusted for covariates. Yoghurt consumption (measured from 24-h recall data) was not associated with obesity $(P=0.23)$ in a model adjusted for covariates High yoghurt consumption was associated with a lower risk of overweight/ obesity when compared with low consumption $(P$-value for trend $<0.001)$. The significance was for all yoghurt and whole fat yoghurt but not low fat Odds of overweight participants developing obesity reduced (NS) with increased consumption of yoghurt, controlling for confounders

Abbreviations: BMI, body mass index; NR, not reported; NS, nonsignificant; RCT, randomised controlled trial; WC, waist circumference.

Baseline anthropometrics

Seven studies included normal-weight or borderline overweight participants. ${ }^{28,33-36,38,44}$ Nine studies were in overweight ${ }^{25,27,37}$ or obese participants, ${ }^{39,41}$ or overweight/obese participants. ${ }^{26}$ Six studies did not clearly report baseline anthropometric data.

\section{Percentage males}

Where reported (19 studies), the sex ratio varied largely between studies. Five studies recruited females only. ${ }^{26,27,29,42,43}$ One crossover trial recruited males only. ${ }^{33}$ In Mozaffarian et al., ${ }^{36}$ the NHSI and NHSIl cohorts were all female and the HPFS cohort was all male, all pooled for analysis. ${ }^{36}$ In 12 studies of mixed populations, the percentage of male participants ranged from $\sim 16 \%{ }^{31}$ to $55 \%{ }^{38}$

Interventions and comparators

The detailed study methods for all studies are presented in the Supplementary File. The intervention was generally described as 'yoghurt', although some RCTs and cross-over trials specified the yoghurt brand (e.g. Yoplait, Kraft, Dannon). Population-based cohort studies and cross-sectional studies often used food 
frequency questionnaires, recall data or dietary records to ascertain yoghurt consumption. Across the different food frequency questionnaires, the interpretation of 'yoghurt' is likely to be different, and it is possible that probiotic yoghurts may have been included. Definitions of yoghurt consumption levels varied across studies: high consumption was at least seven times a week in Martinez-Gonzalez et al., ${ }^{34}$ but only three servings a week in Gugger et al. $^{34,42}$ In the RCTs, doses of yoghurt varied between at least four times per week ${ }^{31}$ and $1 \mathrm{~L}$ of yoghurt daily. ${ }^{28}$ The crossover trials reported similar doses of yoghurt during the intervention period: $16 \mathrm{oz}(454 \mathrm{~g})$, daily; ${ }^{33} 8 \mathrm{oz}(227 \mathrm{~g})$, three times a day. ${ }^{32}$

Within the study types, comparators varied widely and included other dairy products, dietary supplements, placebo food products or no intervention (i.e. low or no yoghurt, or non-consumers). Unless otherwise specified, the comparator dose was the same as or similar to the intervention dose within a study. Where a variety of foods were potential comparators (e.g. food frequency questionnaires), we defined a suitable comparator as that which was most similar to yoghurt: milk and/or milk drinks.

Four of the six RCTs and the controlled trial reported that an energy-restricted diet and/or physical activity change was adopted by both the treatment and control groups for the duration of the trial. One cross-over trial, McNamara et al., ${ }^{33}$ specified that participants stayed on a controlled low-fat, lowcholesterol diet. Two RCTs investigated the effect of consuming yoghurt around the time of exercise. ${ }^{27,29}$

\section{Quality assessment}

The detailed quality assessment is presented in the Supplementary File. Two RCTs had a low risk of bias in the summary assessment. ${ }^{27,30}$ The remaining five RCTs/CT had a high risk of bias, due to non-completers, problems with randomisation and conflicts of interest. Al-Naggar et al. ${ }^{25}$ was poorly reported, such that the results were questionable and the authors' responses to queries were unclear: the results of the paper were therefore excluded from the systematic review. ${ }^{25}$ The summary quality assessment for both cross-over trials was a high risk of bias due to incomplete data ${ }^{32}$ and funders with conflict of interests. ${ }^{33}$

The prospective cohort study by Martinez-Gonzalez et $a l^{34}$ had a low risk of bias. All of the retrospective cohort studies had an unclear risk of bias. They did not provide sufficient information to determine whether groups were comparable on confounding variables. However, we note that the studies were not designed to provide comparable groups and that four of the five adjusted for confounding variables. ${ }^{35,37-39}$

The cross-sectional studies' summary quality assessments ranged from 6 to 8 stars out of a maximum of 10 on the adapted Newcastle-Ottawa scale. Although none of the studies received a star for 'sample size justification' or 'non-respondents', this is likely to be a reflection of the retrospective nature of the majority of studies (6/7), where information on non-respondents may not be available. Taking this into account, we report a generally low risk of bias across the seven cross-sectional studies.

\section{Yoghurt consumption and BMI}

Four cross-sectional studies ${ }^{40-43}$ (generally low risk of bias) showed that yoghurt consumers have a significantly lower BMI compared with non-consumers, but two RCTs ${ }^{26,27}$ (one low, one high risk of bias) did not show a significant difference in the decrease in BMI between yoghurt and placebo groups over time (Table 2). An association between high yoghurt consumption and low BMI was reported by one cross-sectional study, ${ }^{39}$ but another cross-sectional study ${ }^{45}$ did not show the trend.

One low risk of bias $\mathrm{RCT}^{27}$ reported significant decreases in $\mathrm{BMI}$ for overweight/obese women following a calorie-deficit diet with resistance training in both the yoghurt and control (isotonic placebo beverage) groups over 16 weeks $(P=0.003)$. There was, however, no significant difference between the groups $(P=0.584)$. One high risk of bias study ${ }^{26}$ also showed a significant decrease in BMI for overweight/obese women in both yoghurt and control groups on an energy-restricted diet and exercise programme over 24 weeks $(P<0.05)$, but there was no difference between groups.

Comparative evidence from four cross-sectional studies was consistent in showing that yoghurt consumers had a significantly lower BMI compared with non-consumers in both sexes ${ }^{39,46}$ and females only. ${ }^{42,43}$ Evidence from one cross-sectional study supports the trend, reporting a negative correlation between yoghurt consumption and BMI in both genders. ${ }^{41}$ Conversely, one cross-sectional study did not show the same trend for yoghurt, but did show it for reduced fat milk. ${ }^{45}$ The cross-sectional studies had a generally low risk of bias and the majority of studies adjusted for confounding factors such as age, baseline weight, diet and lifestyle factors.

\section{Yoghurt consumption and body weight}

One $\mathrm{RCT}^{30}$ (low risk of bias) showed that yoghurt consumption significantly reduced body weight compared with a placebo, but two other RCTs ${ }^{26,27}$ (one low, one high risk of bias) and two crossover trials ${ }^{32,33}$ (high risk of bias) did not find a significant difference (Table 2). An association between high yoghurt consumption and low body weight was reported by one retrospective cohort study (Wang et al., ${ }^{39}$ unclear risk of bias) and one CT (Jordan et al.," ${ }^{31}$ high risk of bias); conversely, a small, significant increase in body weight with yoghurt consumption was reported by one $\mathrm{RCT}^{28}$ (high risk of bias).

Evidence from a low risk of bias $\mathrm{RCT}^{30}$ indicated that when part of an energy-restricted diet for overweight/obese people, yoghurt supplementation can help to significantly reduce body weight compared with a placebo (sugar-free gelatin dessert) over 12 weeks. Conversely, two RCTs involving energy-restricted diets (one at high risk of bias and one at low risk of bias) reported no significant differences between yoghurt and control groups (the comparators were: standard protein diet with no added yoghurt and an isotonic placebo beverage). ${ }^{26,27}$ The forest plot in Figure 2ai indicates greater weight loss in participants consuming yoghurt compared with the comparator group $(-0.99,95 \% \mathrm{Cl}:-2.21,0.23)$, but the effect size was not significant $(P=0.11)$. In a sensitivity analysis, excluding the study with a high risk of bias, ${ }^{26}$ the summary effect size became larger $(-1.58,95 \% \mathrm{Cl}:-2.90,-0.26)$ and reached statistical significance $(P=0.02)$ (Figure 2aii). In both standard analysis and sensitivity analysis, heterogeneity was low for both the fixed-effect and random-effects models ( $14 \%$ and $0 \%$, respectively). The summary effect sizes were similar in the fixed-effect model $(Z=-1.80, P=0.07$ and $Z=-2.34, P=0.02$, respectively).

The two cross-over trials did not find any significant change in body weight between the yoghurt and control (calcium carbonate pills; $2 \%$ non-fermented milk) phases of the trials. Both studies had a high risk of bias, were conducted for short treatment periods and participants were of normal weight, or weight was not reported. Because the mean and s.d. of weight change was not reported for the yoghurt and control groups by either of the studies, meta-analysis was not possible.

High yoghurt consumers gained significantly less weight compared with low yoghurt consumers in one retrospective cohort study (Wang et al., ${ }^{39}$ unclear risk of bias) and a similar, nonsignificant trend was reported in the controlled trial by Jordan et $a l^{31} \mathrm{~A}$ significant association between increasing yoghurt consumption and decreasing weight gain was found in another retrospective cohort study, ${ }^{36}$ mixed results in another ${ }^{38}$ and a nonsignificant effect in the remaining study. ${ }^{35}$ Two RCTs, ${ }^{28,29}$ both with high risk of bias, reported an increase in body weight with yoghurt consumption, with a similar body weight increase also being reported in some, but not all comparator groups. These trials are discussed below. 


\section{a Outcome: Change in body weight}

\section{i. All three RCTs}

\begin{tabular}{|c|c|c|c|c|c|c|c|c|c|c|}
\hline \multirow[b]{2}{*}{ Study or Subgroup } & \multicolumn{3}{|c|}{ Yoghurt } & \multicolumn{3}{|c|}{ Control } & \multicolumn{2}{|r|}{ Mean Difference } & \multirow{2}{*}{$\begin{array}{c}\text { Mean Difference } \\
\text { IV, Random, } 95 \% \mathrm{Cl}\end{array}$} & Risk of Bias \\
\hline & Mean & SD & Total & Mean & SD & Total & Weight & IV, Random, $95 \% \mathrm{CI}$ & & $A B C D E F$ \\
\hline Shilsky & -5.5 & 4.5 & 41 & -5.8 & 4.5 & 35 & $31.1 \%$ & $0.30[-1.73,2.33]$ & & \\
\hline Thomas & -2.6 & 4.5 & 15 & -1.2 & 2.5 & 14 & $19.7 \%$ & $-1.40[-4.03,1.23]$ & & \\
\hline Zemel & -6.63 & 2.55 & 18 & -4.99 & 2 & 16 & $49.3 \%$ & $-1.64[-3.17,-0.11]$ & & \\
\hline Total $(95 \% \mathrm{Cl})$ & & & 74 & & & 65 & $100.0 \%$ & $-0.99[-2.21,0.23]$ & & \\
\hline \multicolumn{9}{|c|}{ Heterogeneity: $\mathrm{Tau}^{2}=0.18 ; \mathrm{Chi}^{2}=2.33, \mathrm{df}=2(\mathrm{P}=0.31) ; \mathrm{I}^{2}=14 \%$} & $\begin{array}{ccccc}1 & 1 & & & 1 \\
-4 & -2 & 0 & 2 & 4\end{array}$ & \\
\hline
\end{tabular}

\section{ii. Sensitivity analysis excluding RCT with a high risk of bias}

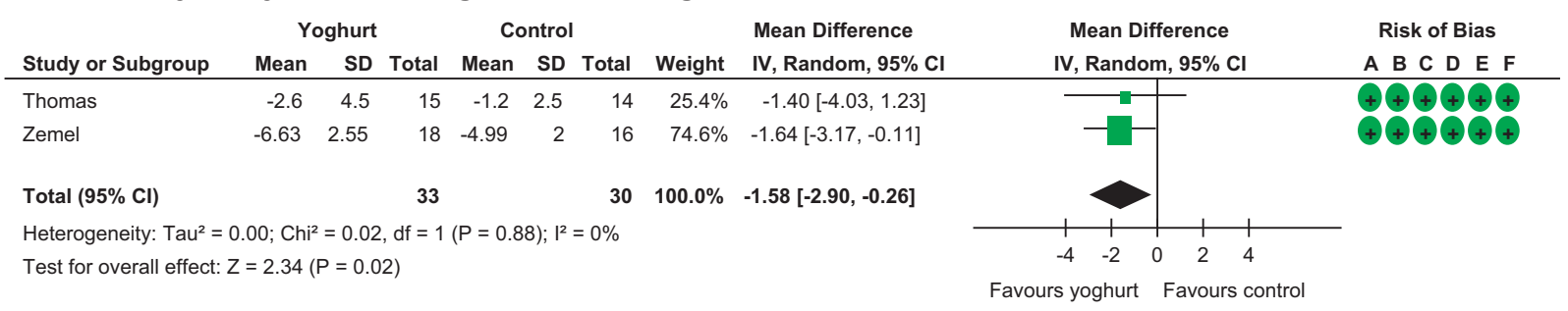

\section{b Outcome: Change in WC}

\begin{tabular}{|c|c|c|c|c|c|c|c|c|c|c|}
\hline \multirow[b]{2}{*}{ Study or Subgroup } & \multicolumn{3}{|c|}{ Yoghurt } & \multicolumn{3}{|c|}{ Control } & \multicolumn{2}{|r|}{ Mean Difference } & \multirow{2}{*}{$\begin{array}{c}\text { Mean Difference } \\
\text { IV, Random, } 95 \% \mathrm{CI}\end{array}$} & Risk of Bias \\
\hline & Mean & SD & Total & Mean & SD & Total & Weight & IV, Random, $95 \% \mathrm{CI}$ & & $A B C D E F$ \\
\hline Shilsky & -4.9 & 3.6 & 41 & -5.2 & 3.7 & 35 & $52.2 \%$ & $0.30[-1.35,1.95]$ & & $?$ \\
\hline Zemel & -3.99 & 2.04 & 18 & -0.58 & 4.16 & 16 & $47.8 \%$ & $-3.41[-5.66,-1.16]$ & & \\
\hline Total $(95 \% \mathrm{Cl})$ & & & 59 & & & 51 & $100.0 \%$ & $-1.47[-5.11,2.16]$ & & \\
\hline \multicolumn{9}{|c|}{ Heterogeneity: $\mathrm{Tau}^{2}=5.87 ; \mathrm{Chi}^{2}=6.81, \mathrm{df}=1(\mathrm{P}=0.009) ; \mathrm{I}^{2}=85 \%$} & $\begin{array}{cccc} & 1 & & \\
-4 & -2 & 0 & 2\end{array}$ & \\
\hline
\end{tabular}

\section{Outcome: Change in body fat}

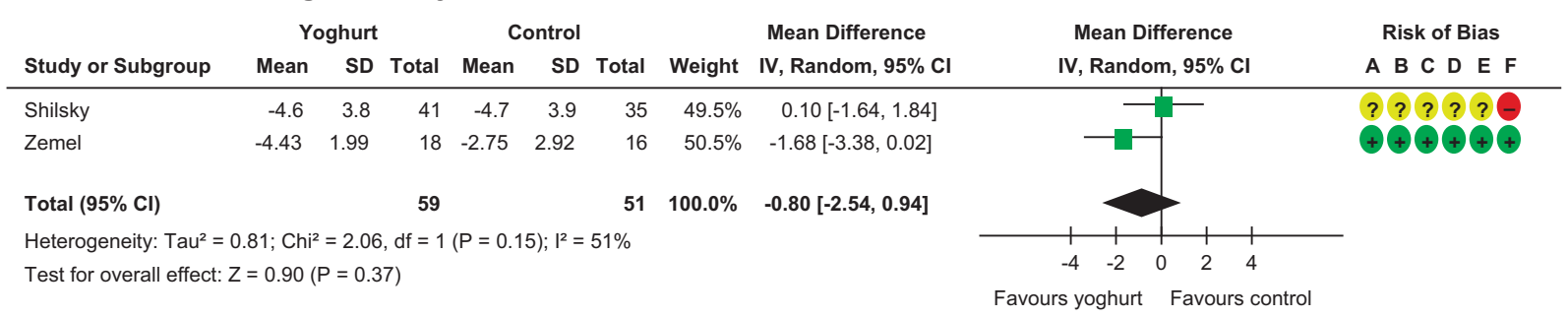

Figure 2. Forest plot of comparison: yoghurt vs control as part of an energy-restricted diet in mostly overweight/obese participants. Risk of bias: (A) random sequence generation; (B) allocation concealment; (C) blinding of participants and personnel; (D) incomplete outcome data; (E) selective reporting; (F) other bias. (a) Outcome: change in body weight. (i) All three RCTs. (ii) Sensitivity analysis excluding RCT with a high risk of bias. (b) Outcome: change in WC. (c) Outcome: change in body fat.

Yoghurt consumption and WC

One $\mathrm{RCT}^{30}$ (low risk of bias) and three cross-sectional studies (low risk of bias) indicated that consumers of yoghurt had significantly lower WC compared with control/non-consumers of yoghurt (Table 2). Conversely, two RCTs ${ }^{26,27}$ (one low, one high risk of bias) did not find a significant difference. A significant association between high yoghurt consumption and low WC was reported by two cohort studies ${ }^{38,39}$ (for overweight men only, in one of these) and two cross-sectional studies. ${ }^{45,46}$ One cohort study ${ }^{35}$ showed a significant positive effect.

One low risk of bias $\mathrm{RCT}^{30}$ showed a significant decrease in the WC of overweight/obese people consuming yoghurt compared with a control group (sugar-free gelatin dessert) after 12 weeks on an energy-restricted diet. In contrast, one low ${ }^{27}$ and one high risk of bias ${ }^{26}$ RCT of overweight/obese people on energy-restricted diets reported no difference between groups. Shilsky et al. ${ }^{26}$ and
Zemel et al. ${ }^{30}$ provided data that could be pooled (Figure $2 \mathrm{~b}$ ). The summary effect size indicated a nonsignificant benefit for yoghurt over control in terms of WC: $-1.47(95 \% \mathrm{Cl}$ : $5.11,2.16)(P=0.43)$. Because of the high risk of bias of Shilsky et al., ${ }^{26}$ the summary effect should be interpreted with caution. The summary effect size was not significant for the fixed-effect model $(Z=-1.47 ; P=0.14)$, and heterogeneity was considerable $\left(I^{2}=85 \%\right)$ for both the fixedeffect and random-effects models, suggesting caution when interpreting the summary effect size. This heterogeneity may be because of a difference in participant numbers between studies, different comparators or differences in participants at baseline (sex, ethnicity).

Three low risk of bias cross-sectional studies reported that yoghurt consumers had significantly smaller WC compared with non-consumers (both sexes together: Wang et al.;6 both sexes separately: Albertson et al.;0 females only: Joshi et al. ${ }^{43}$ ). 
Two cohort studies provided evidence for a negative association between yoghurt consumption and WC in overweight people over a follow-up period of 6 or 12.9 years, but did not provide a direct comparison between yoghurt and a control. ${ }^{38,39}$ A positive association between low-fat yoghurt and WC was reported in another small cohort study, but there was a lack of correction for other diet factors in the analysis. ${ }^{35}$ A significant negative association between yoghurt consumption and WC for both sexes together was reported by three cross-sectional studies. ${ }^{40,41,45}$ The cohort and most cross-sectional studies adjusted for confounding factors such as age, baseline weight, diet and lifestyle factors.

\section{Yoghurt consumption and body fat}

One RCT (low risk of bias) and one cross-sectional study (moderate risk of bias) indicated that consumers of yoghurt had significantly lower body fat compared with a control group (Table 2). Conversely, three RCTs (one low, two high risk of bias) did not find a significant difference.

Evidence from one low risk of bias $\mathrm{RCT}^{30}$ indicated that yoghurt consumption can significantly decrease the amount of body fat and trunk fat of overweight/obese people compared with no yoghurt consumption over 12 weeks when on an energyrestricted diet.

One low $^{27}$ and two high risk of bias ${ }^{26,29}$ RCTs reported no significant difference between yoghurt and control groups in primarily overweight/obese yoghurt consumers, on a caloriedeficit diet and/or resistance training. Comparator groups were an isotonic placebo beverage, ${ }^{27}$ no added yoghurt and standard protein $^{26}$ and carbohydrate only or carbohydrate and protein. ${ }^{29}$

The forest plot in Figure 2c compares the change in body fat between participants consuming yoghurt compared with a control from the studies that were similar enough to be combined. ${ }^{26,30}$ All participants were on an energy-restricted diet and were either overweight or obese at baseline. The summary effect size showed no benefit for yoghurt over the comparator in terms of change in body fat: $-0.80(95 \% \mathrm{Cl}:-2.54,0.94)$. Shilsky et al. $^{26}$ had a high risk of bias, thus the summary effect must be viewed with caution. The summary effect size was not significant for the fixed-effect model $(Z=-1.31 ; P=0.19)$. Heterogeneity was on the borderline of moderate and substantial $\left(I^{2}=51 \%\right)$ for both the fixed-effect and random-effects models, suggesting caution should be exercised in the interpretation of the summary effect size. This heterogeneity may be because of differences in participant numbers, baseline characteristics and comparators.

The cross-sectional study by Joshi et al. ${ }^{43}$ (moderate risk of bias) reported that female yoghurt consumers had significantly less body fat compared with non-yoghurt consumers; another crosssectional study reported significant inverse associations between yoghurt consumption and body fat and abdominal fat, ${ }^{45}$ whereas a cohort study did not find an association. ${ }^{35}$

Yoghurt consumption and lean body mass

One $\mathrm{RCT}^{30}$ (low risk of bias) reported that consumers of yoghurt had lost significantly less lean body mass compared with a control group (Table 2). Conversely, two RCTs (one low, ${ }^{27}$ one high risk of bias $^{29}$ ) did not find a significant difference.

Evidence from one RCT, ${ }^{30}$ which was at low risk of bias shows that when on a calorie-restricted diet, overweight/obese consumers of yoghurt lost significantly less lean body mass compared with the control participants (overweight/obese non-consumers of yoghurt) over 12 weeks. In contrast, there was no difference in the percent lean body mass increase observed in both yoghurt and control groups in the two RCTs that incorporated resistance training into the trial. ${ }^{27,29}$ One trial in overweight/obese people, used an isotonic placebo beverage as the comparator, reported after 8 weeks and was at low risk of bias. ${ }^{27}$ The other study did not report the body weight of the study population, provided carbohydrate only or carbohydrate and protein as the comparator, reported at 16 weeks and was at high risk of bias. ${ }^{29}$ The studies could not be pooled in a meta-analysis because of differences in outcome reporting and involvement in diets.

Yoghurt consumption and risk/proportion of overweight/obesity One large prospective cohort study (low risk of bias) provided comparative evidence that high consumers of yoghurt who were not overweight at baseline had a significantly lower risk of overweight/obesity compared with low consumers (Table 2). ${ }^{34}$ A retrospective cohort study found a nonsignificant reduction in the odds of overweight people becoming obese with increasing yoghurt consumption. ${ }^{37}$

Evidence from two comparative cross-sectional studies (one at low risk of bias and one at moderate risk of bias) showed that yoghurt consumption was associated with lower risk or prevalence of overweight/obesity compared with low or no yoghurt consumption in females. ${ }^{40,42}$ One cross-sectional study had conflicting results depending on how yoghurt intake was measured, ${ }^{44}$ and another showed a negative association between yoghurt consumption and both obesity and central obesity. ${ }^{41}$

Most of the cohort and cross-sectional studies adjusted for confounding factors such as age, baseline weight, diet and lifestyle factors.

\section{DISCUSSION}

The aim of this review was to examine the available evidence on the effects of conventional yoghurt consumption on weightrelated outcomes. We found evidence from studies comparing yoghurt to other interventions supporting an association between yoghurt consumption and lower BMI, lower body weight/weight gain, smaller WC and lower body fat in normal-weight, overweight and obese people and/or the general population. We did not find evidence of causality.

Only one of five RCTs reported significant favourable weightrelated outcomes for yoghurt compared with the control group, although pooling of data from other RCTs supported the effect for body weight. Control group data from RCTs indicated that energyrestricted diets with/without resistance training may produce more favourable weight-related outcomes (body weight, WC, body fat and lean body mass) for overweight/obese people, rather than the yoghurt supplementation itself. We recognise that the RCTs were small sized, and generally focused on overweight/ obese people and energy-restricted diets with/without exercise, limiting the generalisability of the outcomes from these studies. Two RCTs ${ }^{28,29}$ reported an increase in body weight with yoghurt consumption, with a similar body weight increase also being reported in some, but not all comparator groups. One of these trials ${ }^{28}$ did not have weight loss as an outcome and gave a supplement of $1 \mathrm{~L}$ of several dairy products in addition to the participants' usual dietary intakes. This extra dairy intake may be an explanation for the observed weight gain. In the second $\mathrm{RCT}^{29}$ the primary outcome was also not weight loss and participants were exercising. They had a $1.1 \mathrm{~kg}$ rise in body weight, but a rise in fat-free mass of $2 \mathrm{~kg}$ and a loss in fat mass of $0.9 \mathrm{~kg}$, suggesting that there is a shift from fat mass to lean mass. ${ }^{47,48}$

High yoghurt consumption was associated with a lower risk of overweight/obesity compared with low yoghurt consumption. This trend varied with race in some studies: it was not seen in Asian people when using 24-h recall data (participants in one cross-sectional study ${ }^{44}$ ), or in non-Hispanic Black populations (subgroup analysis in one study ${ }^{41}$ ). Again, because of the potential for residual confounding within these observational studies, this finding should be interpreted with caution. 
Limitations of the evidence

We found poor reporting and an absence of detail in many reports and when contacting authors directly, which limited our ability to compare studies and also our ability to assess the methodological quality of studies. Only one study reported the bacterial strain of yoghurt. Particularly in cohort or cross-sectional studies, which often used questionnaires or food diaries to ascertain yoghurt consumption, different definitions and interpretations of 'yoghurt' may have introduced some noise into the data, for example, probiotic yoghurt may have been included. None of the studies was prospectively registered in ClinicalTrials.gov or the registers accessed via the International Clinical Trials Registry Platform. This might be seen as an indicator of risk of bias, but we note that currently few systematic reviews could be completed based on registered trials alone. ${ }^{49}$

Yoghurt consumption is highly likely to be linked to healthy eating and lifestyle attributes, which are, in turn, likely to be linked to positive weight-related outcomes (i.e. lower BMI), creating a systematic bias. Very often, several of these attributes occur together in individuals, inflating the confounding effect. Confounders important for this topic include, but are not limited to: socioeconomic status; energy intake; diet quality; physical activity levels; body weight at baseline; gender and race. Some, but not all of the potential confounders were added as covariates in analyses by the included studies, indicating that residual confounding is likely to remain. For example, socioeconomic status is likely to be a major confounder, but it was only reported by half of the cohort and less than half of the cross- sectional studies.

The studies retrieved for this review were heterogeneous in aspects of their study design, populations, treatment groups and outcomes, an expected outcome of including different study types in this review. Because of the differences between studies, there were only a limited number of studies that were suitable for combination into a meta-analysis. Residual variation between the studies that were combined led to heterogeneity within the metaanalysis (as seen in the high $l^{2}$ statistics in summary effect sizes for WC and body fat, although not for body weight), and, in turn, a low confidence in summary estimates.

Although we investigated the possibility of combining data from single arms from studies of different designs that were conducted in similar ways, in a meta-analysis, we found no instances where study arms could be combined, partly because of the lack of comparable study types: there were only two crossover trials.

Because of the high heterogeneity between studies, combining our narrative summaries with an examination of individual study results will give a more complete and reliable reflection of the evidence base.

Sometimes, studies did not report comparative data (i.e. between yoghurt and a comparator, or yoghurt and low/no yoghurt groups). This information is key in separating the effects of treatment group and the lack of this information limited our ability to draw robust conclusions from the studies. Because not all studies reported comparative data, we also report noncomparative data. Although this type of data represents a lower strength of evidence, it provides additional information to support conclusions drawn from comparative data.

\section{Limitations of this review}

In any review of evidence, there is a potential for publication bias, where the body of published evidence is not representative of the studies that have been conducted in a particular topic area. There were not enough comparable studies (with similar outcome measures and timescales) to reliably assess the potential for publication bias. Until more studies in this topic are available, we must assume that there is a potential for this review to be influenced by publication bias to an unknown extent.
Recommendations for future research

We recommend that future experimental studies addressing this question use standard, non-probiotic yoghurt cultures, define the type of yoghurt and the cultures included and use amounts of yoghurt similar to those consumed in the general population. Predefined or post hoc analyses of subgroups (e.g. race, gender, socioeconomic class) could be undertaken separately. In particular, because of the potential for gender-related bias in yoghurt consumption (identified by studies in this review), both sexes should be included, and either analysed separately or gender added as a covariate in analysis.

In future studies, the comparator should be no yoghurt consumption with yoghurt replaced by an isocaloric replacement food, to separate the effect of yoghurt. In cases where using this comparator may compromise blinding, an appropriate placebo product, such as a gelatin-based dessert, could be used as a comparator. Another study design could use two distinct interventions, with each intervention acting as the other's control.

Future studies should be powered appropriately to maximise the likelihood of detecting a causal effect. Cohort and crosssectional studies can enable an assessment of the outcome effect in the 'real-world' context, including and controlling for confounders in analyses. Blinded or placebo-controlled RCTs in a community-based setting, controlling for other diet and lifestyle factors (e.g. a normal, stable diet and exercise regime) may be able to separate the treatment effect of yoghurt, although the controls may not be representative of yoghurt consumers in the general population. Whatever the study type, full measurement, inclusion and reporting the impact of potential confounders in analyses are imperative in all future studies, because of their high potential importance in this area.

We recommend that studies include and report comparative analyses between intervention groups, maximising the availability of combinable data for future meta-analysis.

Future studies in this topic should measure the following outcomes: BMI, body weight, \% body fat, \% lean body mass and WC, and measurements should be undertaken by health professionals, rather than self-reported, to minimise error. We recommend that, to show meaningful (long term and stable) weight-related outcomes, the minimum duration of yoghurt intervention should be 12 months. However, shorter term studies, such as 3 months, can be a useful proof of principle.

We recommend that any future studies be registered (e.g. in ClinicalTrials.gov) and carefully planned to minimise their risk of bias, so that they may be suitable for combination in metaanalysis. Examples of studies with a low risk of bias are the RCTs by Thomas et al. ${ }^{27}$ and Zemel et al., ${ }^{30}$ the prospective cohort study by Martinez-Gonzalez et $a l^{34}$ and most of the cross-sectional studies.

We recommend that published study reports should provide details that would enable reviewers to extract data, assess the methodological quality of the study and maximise the potential for inclusion in meta-analyses. The specific information required will vary between study types, but the following areas are where we have experienced a lack of information:

- Basic study information such as country of study, study dates and whether the study is prospective or retrospective.

- Inclusion and exclusion criteria.

- Baseline population characteristics (by intervention group), including age, sex, baseline weight-related outcomes measures, ethnicity, prognostic and confounding factors (e.g. socioeconomic status, diet and exercise levels).

- Intervention and comparator details including dosing regimen and the strains and type of yoghurt used.

- Full details of analysis methods and justification for sample sizes used.

- Follow-up times. 
- Numbers of non-respondents or dropouts by intervention group, with reasons for dropout.

- Outcome data as means and s.d.s or odds ratios.

- Comparative data from analyses, including $P$-values.

We also recommend that future studies provide full details of the adjustment for confounders in the methods section of their reports. This should include detailing the rationale behind including the potential confounders, how the data was collected, how it was inputted as a covariate in the analysis and whether the analysis showed any association between yoghurt consumption and the confounder. The current literature is dominated by studies from the United States of America, and we suggest that more studies be conducted in European countries, where yoghurt has been more traditionally consumed.

\section{CONCLUSIONS}

There is evidence to suggest that yoghurt consumption is associated with lower BMI, lower body weight/weight gain, smaller WC and lower body fat in a mix of normal-weight, overweight and obese people and/or the general population. We acknowledge the limitations that stem from heterogeneous study designs, small sizes of RCTs, the uncertainties around confounding and the inclusion of non-comparative data and unclear/moderate/high risk of bias studies. The data therefore suggests a role for yoghurt in weight management, but cannot determine a cause-effect relationship. We conclude that the evidence in the literature from crosssectional and cohort studies is sufficiently positive that additional studies of this type will be superfluous. Well-designed, RCTs with adequate numbers for sufficient power are needed to get a better understanding of the possible mechanisms of action and the plausible cause-effect relationships. Consumption of yoghurt will not be a panacea for overweight/obesity, but the simple addition of yoghurt to the daily diet may facilitate significant loss of body fat. This relatively achievable and low-cost dietary change could thereby help in minimising the impact of obesity and improve public health.

The potential of yoghurt for improving body weight/composition calls for increasing funding for research in this area.

\section{CONFLICT OF INTEREST}

York Health Economics Consortium received funding from Danone Institute International to conduct this review. York Health Economics Consortium has received funding from the Global Alliance for Probiotics, Danone and the European Food Safety Agency for projects involving food and health topics. FJK is member of the Scientific Advisory Board Global Dairy Platform, Chicago, IL, USA. RS is President of Danone Institute International. ILW is employed by the Danone Company, France.

\section{ACKNOWLEDGEMENTS}

We are grateful for assistance in data extraction and checking from Donna Coffin. This project was funded by Danone Institute International.

\section{REFERENCES}

1 Bonjour JP, Benoit V, Payen F, Kraenzlin M. Consumption of yogurts fortified in vitamin $\mathrm{D}$ and calcium reduces serum parathyroid hormone and markers of bone resorption: a double-blind randomized controlled trial in institutionalized elderly women. J Clin Endocrinol Metab 2013; 98: 2915-2921.

2 Douglas SM, Ortinau LC, Hoertel HA, Leidy HJ. Low, moderate, or high protein yogurt snacks on appetite control and subsequent eating in healthy women. Appetite 2013; 60: 117-122.

3 Codex Committee on Milk and Milk Products. Codex Standard for Fermented Milks (CODEX STAN 243-2003). Codex Alimentarius: New Zealand, 2010, p 11.

4 Meyer AL, Elmadfa I, Herbacek I, Micksche M. Probiotic, as well as conventional yogurt, can enhance the stimulated production of proinflammatory cytokines. J Hum Nutr Diet 2007; 20: 590-598.
5 Burns AA, Livingstone MBE, Welch RW, Lindmark L, Rowland IR. Confirmation of reduced energy intake after consumption of yoghurt containing Olibra. Proc Nutr Soc [Internet] 1998; 57:152a; Available at: http://onlinelibrary.wiley. com/o/cochrane/clcentral/articles/535/CN-00359535/frame.html.

6 O'Connor LM, Lentjes MA, Luben RN, Khaw KT, Wareham NJ, Forouhi NG. Dietary dairy product intake and incident type 2 diabetes: a prospective study using dietary data from a 7-day food diary. Diabetologia 2014; 57: 909-917.

7 Buyuktuncer Z, Fisunoglu M, Guven GS, Unal S, Besler HT. The cholesterol lowering efficacy of plant stanol ester yoghurt in a Turkish population: a doubleblind, placebo-controlled trial. Lipids Health Dis 2013; 12: 91.

8 Kurahashi N, Inoue M, Iwasaki M, Sasazuki S, Tsugane AS. Dairy product, saturated fatty acid, and calcium intake and prostate cancer in a prospective cohort of Japanese men. Cancer Epidemiol Biomarkers Prev 2008; 17: 930-937.

9 Ballesta S, Velasco C, Borobio MV, Arguelles F, Perea EJ. Fresh versus pasteurized yogurt: comparative study of the effects on microbiological and immunological parameters, and gastrointestinal comfort. Enferm Infecc Microbiol Clin 2008; 26: 552-557.

10 Pashapour N, lou SG. Evaluation of yogurt effect on acute diarrhea in 6-24-month-old hospitalized infants. Turk J Pediatr 2006; 48: 115-118.

11 Sazawal S, Habib A, Dhingra U, Dutta A, Dhingra P, Sarkar A et al. Impact of micronutrient fortification of yoghurt on micronutrient status markers and growth - a randomized double blind controlled trial among school children in Bangladesh. BMC Public Health 2013; 13: 514.

12 Goldbohm RA, Chorus AMJ, Garre FG, Schouten LJ, Brandt PA. Dairy consumption and 10-y total and cardiovascular mortality: a prospective cohort study in the Netherlands. Am J Clin Nutr 2011; 93: 615-627.

13 Aune D, Norat T, Romundstad P, Vatten LJ. Dairy products and the risk of type 2 diabetes: a systematic review and dose-response meta-analysis of cohort studies. Am J Clin Nutr 2013; 98: 1066-1083.

14 Gao D, Ning N, Wang C, Wang Y, Li Q, Meng Z et al. Dairy products consumption and risk of type 2 diabetes: systematic review and dose-response meta-analysis. PLoS One [electronic resource] 2013; 8: e73965.

15 Tong X, Dong JY, Wu ZW, Li W, Qin LQ. Dairy consumption and risk of type 2 diabetes mellitus: a meta-analysis of cohort studies. Eur J Clin Nutr 2011; 65: 1027-1031.

16 Aune D, Lau R, Chan DSM, Vieira R, Greenwood DC, Kampman E et al. Dairy products and colorectal cancer risk: a systematic review and meta-analysis of cohort studies. Ann Oncol 2012; 23: 37-45.

17 Soedamah-Muthu SS, Verberne LD, Ding EL, Engberink MF, Geleijnse JM. Dairy consumption and incidence of hypertension: a dose-response meta-analysis of prospective cohort studies. Hypertension 2012; 60: 1131-1137.

18 O'Sullivan TA, Hafekost K, Mitrou F, Lawrence D. Food sources of saturated fat and the association with mortality: a meta-analysis. Am J Public Health 2013; 103: e31-e42.

19 Higgins J, Green S (eds). Cochrane Handbook for Systematic Reviews of Interventions, Version 5.1.0 (updated March 2011). The Cochrane Collaboration, 2011.

20 Liberati A, Altman D, Tezlaff J, Mulrow C, Gotzsche P, loannidis J et al. The PRISMA statement for reporting systematic reviews and meta-analyses of studies that evaluate healthcare interventions: explanation and elaboration. BMJ 2009; 339: b2700.

21 Khan KK, ter Riet G, Popay J, Nixon J, Kleijnen J. Conducting the review, 5: Study Quality Assessment. In: CRD (ed), Systematic Reviews: CRD's Guidance for Udertaking Reviews in Health Care [Internet]. University of York: York, UK, 2009.

22 Wells GA, Shea B, O'Connell D, Peterson J, Welch V, Losos M et al. The Newcastle-Ottawa Scale (NOS) for Assessing the Quality of Nonrandomised Studies in Meta-analyses [web page]. Ottawa Hospital Research Institute: Ottawa, Canada, 2014; Available at: http://www.ohri.ca/programs/clinical_epidemiology/ oxford.asp.

23 Lee $Y$, Shin H, Yon M, Koh E, Lee JY, Kim DH et al. Dairy product intake is associated with the risk of obesity and abdominal obesity in middle-aged Koreans. Obesity Facts 2012; 5: 28-29.

24 Sorenson AW, Pfister R, Smith K, Delhumeau C. Identifying the role of milk and milk products on nutritional and health issues for adults fifty years of age and older. FASEB J 2004; 18: abstract 582.5.

25 Al-Naggar RA, Osman MT, Abdulghani M. Low fat yogurt reduce weight: randomized clinical trial from Malaysia. Res J Pharm Biol Chem Sci 2014; 5: 593-597.

26 Shlisky JD, Durward CM, Zack MK, Campbell JK, Jonnalagadda SS, Gugger C et al. Effects of an energy-restricted, moderate-protein diet plan with non-fat dairy on weight changes: 12 weeks of weight loss (WL) followed by 12 weeks of WL maintenance. FASEB J 2012; 26.

27 Thomas DT, Wideman L, Lovelady CA. Effects of a dairy supplement and resistance training on lean mass and insulin-like growth factor in women. Int J Sport Nutr Exerc Metab 2011; 21: 181-188. 
28 Thompson LU, Jenkins DJ, Amer MA, Reichert R, Jenkins A, Kamulsky J. The effect of fermented and unfermented milks on serum cholesterol. Am J Clin Nutr 1982; 36: $1106-1111$.

29 White KM, Bauer SJ, Hartz KK, Baldridge M. Changes in body composition with yogurt consumption during resistance training in women. Int J Sport Nutr Exerc Metab 2009; 19: 18-33.

30 Zemel MB, Richards J, Mathis S, Milstead A, Gebhardt L, Silva E. Dairy augmentation of total and central fat loss in obese subjects. Int J Obes 2005; 29: 391-397.

31 Jordan HA, Levitz LS, Utgoff KL, Lee HL. Role of food characteristics in behavioral change and weight loss. J Am Diet Assoc 1981; 79: 24-29.

32 Bazzarre TL, Wu S-ML, Yuhas JA. Total and high density lipo protein cholesterol concentrations following yoghurt and calcium supplementation. Nutr Rep Int 1983; 28: 1225-1232.

33 McNamara DJ, Lowell AE, Sabb JE. Effect of yogurt intake on plasma lipid and lipoprotein levels in normolipidemic males. Atherosclerosis 1989; 79: 167-171

34 Martinez-Gonzalez MA, Sayon-Orea C, Ruiz-Canela $M$, de la Fuente $C$, Gea A, Bes-Rastrollo M. Yogurt consumption, weight change and risk of overweight/obesity: The SUN cohort study. Nutr Metab Cardiovasc Dis 2014; 24: 1189-1196.

35 Drapeau V, Despres JP, Bouchard C, Allard L, Fournier G, Leblanc C et al. Modifications in food-group consumption are related to long-term body-weight changes. Am J Clin Nutr 2004; 80: 29-37.

36 Mozaffarian D, Hao T, Rimm EB, Willett WC, Hu FB. Changes in diet and lifestyle and long-term weight gain in women and men. N Engl J Med 2011; 364: 2392-2404.

37 Pereira MA, Jacobs DR Jr, Van Horn L, Slattery ML, Kartashov Al, Ludwig DS. Dairy consumption, obesity, and the insulin resistance syndrome in young adults: the CARDIA Study. JAMA 2002; 287: 2081-2089.

38 Vergnaud AC, Peneau S, Chat-Yung S, Kesse E, Czernichow S, Galan P et al. Dairy consumption and 6-y changes in body weight and waist circumference in middle-aged French adults. Am J Clin Nutr 2008; 88: 1248-1255.

39 Wang H, Troy LM, Rogers GT, Fox CS, McKeown NM, Meigs JB et al. Longitudinal association between dairy consumption and changes of body weight and waist circumference: the Framingham Heart Study. Int J Obes 2014; 38: 299-305.

40 Albertson AM, Holschuh NM, Eldridge AL. Yogurt consumption in the United States: effect on nutrient intakes and body measures in adults 19+ years. FASEB J 2007; 21: A1061.
41 Beydoun MA, Gary TL, Caballero BH, Lawrence RS, Cheskin L, Wang Y. Ethnic differences in dairy and related nutrient consumption among US adults and their association with obesity, central obesity, and the metabolic syndrome. Am J Clin Nutr 2008; 87: 1914-1925.

42 Gugger C, Joshi N, Albertson A. Yogurt consumption is associated with lower body weight status and improved nutrient intakes in adult women. FASEB $J$ 2014; 1.

43 Joshi NA, Albertson AM, Bell E. Yogurt intake is associated with favorable nutrient intake and healthy body measures in US women: Results from NHANES 2007-08. FASEB J 2011; 25.

44 Lee H-J, Cho J-I, Lee H-SH, Kim C-I, Cho E. Intakes of dairy products and calcium and obesity in Korean adults: Korean National Health and Nutrition Examination Surveys (KNHANES) 2007-2009. PLoS One [electronic resource] 2014; 9: e99085.

45 Murphy KJ, Crichton GE, Dyer KA, Coates AM, Pettman TL, Milte C et al. Dairy foods and dairy protein consumption is inversely related to markers of adiposity in obese men and women. Nutrients 2013; 5: 4665-4684.

46 Wang $\mathrm{H}$, Livingston KA, Fox CS, Meigs JB, Jacques PF. Yogurt consumption is associated with better diet quality and metabolic profile in American men and women. Nutr Res 2013; 33: 18-26.

47 Beijers HJ, Ferreira I, Bravenboer B, Henry RM, Schalkwijk CG, Dekker JM et al. Higher central fat mass and lower peripheral lean mass are independent determinants of endothelial dysfunction in the elderly: the Hoorn study. Atherosclerosis 2014; 233: 310-318.

48 De Lorenzo A, Bianchi A, Maroni P, lannarelli A, Di Daniele N, lacopino L et al. Adiposity rather than BMI determines metabolic risk. Int J Cardiol 2013; 166: 111-117.

49 Glanville JM, Duffy S, McCool R, Varley D. Searching ClinicalTrials.gov and the International Clinical Trials Registry Platform to inform systematic reviews: what are the optimal search approaches? J Med Libr Assoc 2014; 102: 177-183.

(c) (i) $€$ This work is licensed under a Creative Commons AttributionNonCommercial-NoDerivs 4.0 International License. The images or other third party material in this article are included in the article's Creative Commons license, unless indicated otherwise in the credit line; if the material is not included under the Creative Commons license, users will need to obtain permission from the license holder to reproduce the material. To view a copy of this license, visit http:// creativecommons.org/licenses/by-nc-nd/4.0/

Supplementary Information accompanies this paper on International Journal of Obesity website (http://www.nature.com/ijo) 